\title{
Principales influencias de las novelas de space opera en el Perú durante el siglo XXI
}

Main influences of the space opera novels in Peru during the 21 st century

\author{
Carlos Andrés de la Torre Paredes \\ Universidad Nacional Mayor de San Marcos, Lima, Perú \\ carlosandres.delatorre@unmsm.edu.pe \\ ORCID: https://orcid.org/0000-0002-9410-4219
}

\section{Resumen}

El presente artículo tiene como finalidad comprender las principales influencias de las novelas enmarcadas en el subgénero de la ciencia ficción conocido como space opera producido en el Perú durante el siglo XXI. Para tal fin, se analizan 16 obras de siete autores distintos, publicados de 2000 a 2019. Estas obras son estudiadas desde su planteamiento ficcional, como muestra de una tradición literaria que empieza a forjarse en el Perú. Asimismo, se explorarán los lazos comunicantes entre las distintas obras, relacionados, principalmente, con representaciones de la cultura popular con matices generacionales.

Palabras clave Ciencia ficción, space opera, Perú, novela, siglo XXI

\begin{abstract}
This article aims to understand the main influences of novels framed in the subgenre of science fiction known as space opera produced in Peru during the 21st century. For this purpose, sixteen works by seven different authors, published between 2000 and 2019, are analyzed. These works are analyzed from their fictional approach, as a sample of a literary tradition that is beginning to take shape in Peru. Likewise, the communicating ties between the different works will be explored, mainly related to representations of popular culture with generational nuances.
\end{abstract}

Keywords Science fiction, space opera, Peru, novel, XXI century

Fecha de envio: 9/2/2021 Fecha de aceptación: 19/3/2021 


\section{Introducción}

El siglo XXI en el Perú ha traído consigo un auge en la producción y el consumo de ciencia ficción nacional. Sin embargo, es aproximadamente desde 2010 que la ciencia ficción en el país adquiere relevancia editorial, con la aparición de editoriales interesadas en el género y cada vez más obras literarias, muchas de las cuales se valen de referencias de la cultura de masas: el cine, la televisión, las historietas, los animes, los videojuegos, además de otras expresiones difundidas globalmente. Entre estas obras se encuentran varias novelas de space opera, 16 de las cuales analizaremos como un fenómeno particular dentro del universo de la ciencia ficción peruana, a fin de reconocer sus principales características e influencias.

Esto resulta relevante debido a que el subgénero de la space opera es uno de los subgéneros de la ciencia ficción más consumidos a nivel global, y comprender sus características y sus influencias en la producción peruana podría contribuir al debate editorial y académico sobre las posibilidades de la ciencia ficción como expresión artístico-cultural.

La revisión de estas novelas permitirá tener un panorama de la diversidad de referencias y planteamientos que influencian a la space opera peruana del siglo XXI, la cual, a pesar de sus diferencias, sigue priorizando la acción y la aventura como sus principales motores narrativos, y encuentran gran parte de sus influencias y referencias en la cultura de masas.

\section{¿Qué es la space opera?}

Como ya se mencionó, analizaremos un total de 16 novelas peruanas enmarcadas en el subgénero de la space opera. Estas 16 novelas pertenecen a siete autores distintos, publicados en distintos formatos. El criterio de selección ha respondido a la posibilidad de encontrar referencias respecto a la producción de estos autores que puedan servir de fuente en la investigación.

Para comprender este subgénero, primero debemos definir a qué nos referimos cuando hablamos de ciencia ficción. 
Si bien el término se encuentra en permanente debate debido a sus distintas variantes, para fines de esta investigación se tomará en consideración la definición que hace Isaac Asimov en su libro Sobre la ciencia ficción, la cual considera a la ciencia ficción como una de las variantes de la ficción surrealista:

Para distinguir entre las dos variedades principales de la ficción surrea-
lista, yo diría que en el caso de la ciencia ficción el fondo surreal de la
historia podría derivarse de nuestro propio medio a través de los cam-
bios correspondientes en el nivel de la ciencia y la tecnología. El cambio
podría representar un avance, como el desarrollo de colonias en Marte, o
la interpretación de señales provenientes de formas de vida extraterres-
tre. Podría representar un retroceso, como en una descripción de la des-
trucción de nuestra civilización tecnológica por una catástrofe nuclear o
ecológica (Asimov, 1999, p. 8).

Con esto, Isaac Asimov quiere decir que, cuando los elementos constitutivos de la sociedad y la cultura se reproducen de manera alterada debido a la intervención de elementos científico-tecnológicos en las ficciones planteadas, también estamos frente a ciencia ficción.

Para entender esto debemos comprender, en primer lugar, cómo es que la ciencia ficción surge como género ficcional a mediados del siglo XVIII con la publicación de la novela Frankenstein o el moderno Prometeo de la autora inglesa Mary Shelley, en la cual un científico logra dar vida a un muerto valiéndose de la tecnología eléctrica, que empezaba a cambiar el mundo por aquellas épocas.

En el libro Sobre ciencia ficción, Isaac Asimov nos dice que la aparición de Frankenstein no resulta de una casualidad, sino que se vio determinada por los procesos sociales y científico-tecnológicos desarrollados durante esa época, como consecuencia de la segunda Revolución Industrial, luego de la cual la sociedad humana tuvo mayor conciencia del paso del tiempo, debido a los cambios generacionales que empezaban a darse gracias a la nueva tecnología. Antes de las revoluciones industriales, abuelos y nietos tenían vidas similares, necesidades, labores y posibilidades similares; luego de las revoluciones industriales, la vida de los abuelos y los nietos no volvería a ser igual: no trabajarían igual, no vestirían igual ni se transportarían de la misma manera. La tecnología se encargaba de cambiar al mundo y ese cambio por fin era percibido por las personas.

Tomemos, por ejemplo, la primera novela a la que se podría definir como de verdadera ciencia ficción, Frankenstein, de Mary Shelley, publicada en 
1818 en Gran Bretaña, la cuna de la Revolución Industrial. Trataba sobre la creación de vida no a través de la magia o lo sobrenatural, sino por la aplicación racional de técnicas científicas (que la señora Shelley dejó prudentemente sin describir). Y trataba sobre las perniciosas consecuencias que eso tenía. Como todos sabemos, el monstruo que fue creado destruyó a Frankenstein, su creador (Asimov, 1999, p. 57).

Por ello, Asimov considera que el cambio en la vida de las personas mediante los procesos tecnológicos y científicos es lo que determina qué es y qué no es ciencia ficción. Sin embargo, a pesar de que esta definición parezca reducir el espectro de lo que podría abarcar la ciencia ficción, podremos encontrar diversas variantes que representan al género, desde las motivadas por encuentros con alienígenas y viajes en naves espaciales, hasta las que recrean algún momento del pasado para, mediante la modificación de algún aspecto de su tecnología, alterar la realidad contingente.

Por esta razón la ciencia ficción se constituye como un formato de ancha base, con posibilidades ficcionales diversas, las cuales determinan los elementos tecnológicos y científicos que utiliza la ficción e incluso la estética que plantea. Entre estas variantes se encuentra la space opera, el subgénero que interesa a nuestra investigación y cuyas principales características tal vez puedan sintetizarse en lo planteado en el blog Café Anime Lair por el autor Cauah:

La space opera es un subgénero de la ciencia ficción caracterizado por la acción, la aventura y el recurso del espacio profundo como escenario. Suele retomar algunos elementos clásicos de la fantasía heroica, épica y el género western, y los trasporta a un contexto ficticio en donde la racionalidad científica adquiere un papel secundario y la historia se centra en el melodrama y los personajes (Cuauh, 2011).

Respecto a la procedencia del término space opera, The Encyclopedia of Science Fiction, publicada originalmente en 1979 por John Clute y Peter Nicholls, y que en 2011 fue subida, íntegramente, a Internet en su página oficial, dice que fue:

Wilson Tucker, quien en 1941 propuso "ópera espacial" como el término apropiado para el "hilo de nave espacial hacky, molido, apestoso, gastado”. Pronto se aplicó en su lugar a coloridas historias de acción y aventuras de conflictos interplanetarios o interestelares (Clute, Nicholls, Langford y Sleight, 2020 [traducción del autor]). 
Así, podemos afirmar que la space opera u ópera espacial se caracteriza por relatar aventuras espaciales, repletas de acción, en las cuales el uso de armas, las explosiones y las luchas tienen una relevancia determinante. Bajo este formato se encuentran ficciones sobre invasiones, batallas espaciales, exploración y conquista universal, entre varias otras posibilidades, que tienen al cosmos como escenario, y la acción y la aventura como eje fundamental de narración.

En este sentido, resulta interesante recordar lo que Fredric Jameson plantea al hacer su categorización de las fases por las que ha pasado la ciencia ficción, al considerar a las aventuras o "series espaciales" como ficciones enmarcadas dentro de la primera fase, la cual:

deriva de manera más inmediata de la obra de Jules Verne, pero podría quizá estar marcada por la tradición estadounidense creada por Una princesa de Marte [1917] de Edgar Rice Burroughs (Jameson, 2009, p. 122).

Con esto hace hincapié en que si bien el viajar por el espacio es una característica de la space opera, que puede rastrearse hasta Julio Verne, estos viajes, de la forma en que se den, están muy marcados por la acción y la aventura. Características que terminan siendo más relevantes que la plausibilidad científica, pues estas ficciones, por tradición, se han permitido alejarse un poco de los conceptos estrictamente científicos, y se acercan al formato de la fantasía. Un ejemplo claro de esto se encuentra en la saga de películas de Star Wars, en la cual, por lo menos durante su primera generación, los personajes principales contaban con poderes sobrenaturales justificados por algo llamado "la fuerza", que no tenía una explicación científica ni racional; este alejamiento científico busca ser resuelto en la segunda generación de películas, cuando se explica que estos poderes que otorga "la fuerza" se deben la gran acumulación de algo que llaman "midiclorianos", una especie de criaturas microscópicas ubicadas en las células de todos los seres vivos. De esta manera intentan brindar una explicación científica a elementos planteados como místicos en un primer momento.

Respecto a esta característica de las ficciones de la space opera, de ser flexibles ante la plausibilidad científica, George Mann, en The Mammoth Encyclopedia of Science Fiction, comenta lo siguiente:

no se molestan en hacer plausibles sus futuros inventados, y están más preocupados por los componentes de aventura de sus historias y más dispuestos a ir más allá de los reinos de plausibilidad científica para crear efectos espectaculares (Mann, 2001, p. 5 [traducción del autor]). 
También resulta interesante lo que el autor David Olier dice respecto a este subgénero, pues le adjudica otra característica, que trasciende lo literario y responde a variables socioeconómicas y culturales, y es la que estas ficciones suelen enmarcarse en sagas que les permiten expandir sus universos, aunque, en la mayoría de los casos, los primeros libros que publican explorando ese universo no son planteados para continuarse, sino que son "autoconclusivos".

En la ciencia ficción, óperas espaciales especialmente, es muy normal que un mismo autor publique varios libros situados en el mismo universo $[\ldots]$

por mi experiencia he comprobado que la mayoría de los primeros libros de una saga (me refiero al libro número 1 de cada saga por fecha de publicación, no por orden cronológico) suele ser una historia casi cerrada en sí misma (Olier, 2020).

\section{Antecedentes}

En el caso del Perú, la space opera no aparecerá hasta bastante avanzado el siglo XX. Resulta importante mencionar que desde mediados de siglo XIX se escribe ciencia ficción. El género no ha tenido demasiada difusión y se ha mantenido como una estética alejada de la tradición literaria.

Si bien se pueden mencionar autores representativos de la ciencia ficción durante el siglo XIX y el siglo XX como Juan Manuel del Portillo, Clemente Palma, Héctor Velarde y José B. Adolph, y que antes de terminar el siglo XX aparece libro Hiperespacios de Giancarlo Stagnaro, que se enmarca en el subgénero de la space opera, es recién en el siglo XXI cuando la ciencia ficción peruana toma fuerza y empieza una tendencia creciente de publicaciones del género en el circuito literario peruano.

La aparición del blog Ciencia Ficción Perú en 2009, dirigido por el escritor Daniel Salvo, y la organización en 2010 del Primer Congreso de Escritores de Fantasía y Ciencia Ficción Peruana, a cargo de Elton Honores, evento académico institucionalizado en 2020 con 10 ediciones consecutivas, marcaron un hito en la ciencia ficción peruana, pues desde estos espacios se dio visibilidad y se abrió el debate académico sobre la producción peruana. Estos espacios coincidían con el incremento de publicaciones de ciencia ficción en el mercado editorial peruano y un interés cada vez mayor por la crítica académica respecto de estas publicaciones, muchas de la cuales aparecían en fanzines o se presentaban en forma de 
publicaciones de autor. Este es el caso de libro Historias de ciencia ficción (2008), del autor Carlos Enrique Saldívar Rosas, libro que luego de 10 años, en 2018, vio una reedición bajo el sello de Torre de Papel Ediciones. Al igual que Saldívar, varios autores aparecen en los alrededores de esos años, muchos de los cuales siguen vigentes en 2020, con publicaciones constantes.

Otro ejemplos de esta efervescencia de la ciencia ficción es la muestra temática Se venden marcianos (2015), publicada por Ediciones Altazor. Luego aparecerían las antologías críticas llevadas a cabo por José Güich Rodríguez, titulada Universos en expansión. Antología crítica de la ciencia ficción peruana: siglos XIX-XXI (2018) y la más reciente de Elton Honores Fantasmas del futuro (2019). Debe considerarse, además, el hecho de que una editorial transnacional como Planeta apostó por el género al publicar dos libros de cuentos de Alexis Iparraguirre, El inventario de las naves (2016) (que veía su reedición) y El fuego de las multitudes (2017), así como también No somos cazafantasmas (2018) de Juan Manuel Robles y El sol infante (2018) de José Güich Rodríguez.

Entre algunas otras obras que aparecen en este periodo de efervescencia podemos mencionar la saga El planeta olvidado, de Carlos Echevarría, que ya cuenta con cuatro libros. También la novela El camino de los Aegeti (2010), de Jeremy Torres, que a la fecha tiene una secuela; y las novelas Los viejos salvajes (2012) de Carlos de la Torre Paredes y Aphokalipzis. La máquina del tiempo (2015) de Jim Rodríguez, las novelas de Alex Gubbins de Luis T. Moy, entre otros textos. $\mathrm{Al}$ respecto, Elton Honores, en su libro La división del laberinto. Estudios sobre la narrativa fantástica peruana contemporánea (1980-2015), publica un artículo sobre las nuevas publicaciones aparecidas de 2010 a 2015, donde se incluyen a varios de estos autores (Honores, 2017, pp. 147-162).

$\mathrm{Al}$ respecto, Honores dice que en esta nueva fantasía atemporal se muestra la intención de construir un mundo ficcional autónomo y sin referencia directa a una realidad histórica. Esto, al parecer, con la intención de que los universos ficcionales se sostengan por sí mismos. También menciona que, al escribir estos textos, los autores parecen haber elegido un conjunto disímil de líneas de influencia, lo que permite entender sus obras como parte de un fenómeno global. Respecto a las estéticas que subyacen en este tipo de fantasía atemporal, menciona la del videojuego, la de la literatura como entretenimiento, la del Internet y sus "redes" y la de la cultura de masas norteamericana, que tiene a Star Wars como un modelo dominante. A estas estéticas se suman referencias, expresiones y críticas socioculturales, entre las cuales Honores incluye el racismo que emerge de la globalidad, las constantes menciones a gobiernos monárquicos y militares, la representación 
de las familias en crisis y desestructuradas, la fantasía mesiánica de un líder millennial que salve al mundo y la aventura masculina como modelo predominante (Honores, 2017, p. 161).

Entre estos autores, que experimentan con distintos formatos de ciencia ficción, el subgénero de la space opera adquiere relevancia, y generó sagas que han mantenido su vigencia editorial. Ejemplos de esto son las sagas de Elplaneta olvidado de Carlos Echevarría y Herederos del cosmos de Carlos de la Torre Paredes. A estas sagas también puede sumarse Planetas perdidos de Alfredo Dammert, pues todas cuentan con varios libros publicados de 2010 a 2019.

\section{Novelas de space opera en el Perú durante el siglo XXI}

Si bien podemos hallar antecedentes de space opera en el siglo XX con Estremadoyro y Stagnaro, decidimos enfocarnos en las publicaciones del siglo XXI, específicamente en las novelas, para buscar lazos comunicantes entre estas y establecer si en la propuesta del space opera de las primeras dos décadas de 2000 se configuran elementos suficientes para considerarla como una estética generacional. Para esto, agrupamos a los autores cuyas obras analizamos en tres grupos relativos a sus influencias.

\section{Influencia de la cultura popular de mediados del siglo $\mathrm{XX}$}

Si bien los autores que publican en el siglo XXI tienen influencias claras de la época en la cual se ubican, este primer grupo se diferencia de los demás al referir, principalmente, una influencia de la cultura popular de mediados del siglo XX, en cuanto encuentran sus principales referencias en las revistas pulp de la época y en los autores clásicos de ciencia ficción, además de valerse de las series de televisión y las películas contemporáneas resaltantes.

Empezaremos hablando de la autora Adriana Alarco de Zadra (Lima, 1937). Su noveleta El Ojo de Orión (2004), publicada de forma electrónica en el boletín literario de la Comunidad CF del 27 de septiembre de 2004, narra las aventuras de Io y Phbx, quienes se enfrentan al poderoso grupo llamado el Ojo de Orión, que busca controlar, mentalmente, a las personas.

La noveleta trata sobre viajes interplanetarios en búsqueda de un lugar para la vida, ya que el planeta Tierra ha tenido problemas ecológicos, lo que ha ocasionado la desaparición de gran parte de la humanidad. En este sentido se podría decir que El Ojo de Orión maneja una narrativa ecologista, potenciada en un planteamiento dicotómico, en el cual los buenos y los malos no tienen matices. La 
historia narra cómo Io viaja por distintos planetas y ayuda a Phbx a convertirse en emperador de la Tierra, para así derrotar al Ojo de Orión.

Durante su recorrido por estos planetas, confraterniza con especies humanoides con características determinadas y arquetípicas. Esta situación recuerda a la utilizada en la serie de televisión Star Trek, en la cual los tripulantes de la nave Enterprise llegaban a distintos planetas, donde interactuaban con sus habitantes, que solían tener características muy definidas y arquetípicas. Según comenta la autora, en el ciclo de entrevistas realizadas por el Instituto Pedro Peralta y Barnuevo - Océano a escritores de novelas de space opera en el Perú durante el siglo XXI, sus influencias están relacionadas con las historietas que leyó durante su adolescencia, principalmente las de Superman, y a series de televisión como las de Flash Gordon: la cultura de masas representativa de gran parte del siglo XX (Instituto Océano, 2020a). Alfredo Dammert (Lima, 1944) es otro de los escritores que han incursionado en la estética del space opera que encuentra sus principales influencias en la cultura popular de mediados del siglo XX. A la fecha, ha publicado cinco libros que se enmarcan en la space opera, todos bajo el sello de Ediciones Altazor.

El primero, la novela Planetas perdidos (2015), inicia una trilogía en la que Arthur, un profesor universitario de Historia, se ve envuelto en distintas aventuras espaciales para salvar a una extraterrestre llamada Lyanne, de la que se enamora, y que es raptada por un grupo de nativos del planeta Alderán, quienes la necesitan para mejorar la genética de su especie. Alderán es un planeta que ha sido casi devastado por diversos desastres y está dominado por un régimen autoritario, que se impone mediante el uso de la tecnología y la ciencia. Este es el motivo de Arthur para viajar al planeta, donde vive un sinfín de aventuras repletas de acción, sumadas a una cuota de romance.

Entre las cualidades más resaltantes de Arthur, se puede encontrar su amplio conocimiento sobre las distintas culturas que habitaron la Tierra, lo cual le facilita interactuar con la sociedad extraterrestre con la que se encuentra. Este conocimiento, sumado a la disciplina que le brindó su oficio como profesor universitario de Historia, conecta con un pragmatismo casi amoral, que le permite realizar sus objetivos. Al respecto, Hans Rothgiesser nos dice lo siguiente:

Arthur —el personaje principal— es un pragmático. No tiene ningún problema en engañar o en hacerle creer a una nativa de que está enamorado de ella, con tal de conseguir lo que quiere. Esto es algo que Flash 
Gordon o Buck Rogers jamás harían. Esto no quiere decir que sea una mala historia. Por lo contrario, hace todo mucho más interesante. Es una especie de giro de tuerca a un género que ya conocemos bastante bien (Rothgiesser, 2015).

Años luz, la segunda novela de la trilogía, aparece en 2016 y continúa con la historia de Arthur para salvar a su amada, quien vuelve a ser raptada. La acción y la aventura siguen siendo el elemento principal de la narración.

Luego de varios años de intentar, inútilmente, rehacer su vida y olvidar su pasado junto a Lyanne, el protagonista decide salir del planeta e ir en búsqueda de su amada. Nuevamente viajará por planetas desconocidos, donde se encontrará con distintas sociedades extraterrestres con características propias. Respecto a esta novela, una nota de prensa en el diario Correo dice lo siguiente:

Años luz tiene como personaje principal a Arthur, quien luego de vivir plenamente con su amada Lyanne en la Tierra, ella es raptada por seres de otro planeta. Pasan los años y Arthur ha dejado su pasado y rehace su vida, sin embargo no se adapta y emprende un viaje junto a dos amigos para encontrar a la extraterrestre que sigue en sus sueños (Correo, 2017).

Al mantener la misma lógica de la primera novela, en este libro las relaciones socioculturales también resultan relevantes para la narración, pues permitirán diferenciar las características socioculturales de las distintas sociedades con las que el protagonista tiene contacto.

Durante la travesía, Arthur conocerá a nuevas especies alienígenas y se enfrentará en batalla para llegar hasta donde Lyanne. Este vínculo entre especies planetarias es tan fuerte que enfrentará el peligro y nos harán vivir situaciones sociológicas y exóticas rodeadas del amor entre un hombre y una extraterrestre (Correo, 2017).

Retorno a la Tierra, publicado en 2017, es el último libro de la trilogía y pone fin a las aventuras de Arthur en el espacio. La historia inicia con Arthur y Lyanne viviendo en Ragnarel, planeta natal de Lyanne, donde la pareja tendrá que enfrentar nuevos obstáculos a su amor. Esta vez el villano será un exnovio de Lyanne. Estos hechos contribuyen a reforzar el planteamiento romántico que se encuentra en la saga. Al respecto, una nota de prensa en Andina dice lo siguiente: 
Para dar fin a la saga, Retorno a la Tierra (2017), Arthur vive con Lyanne en el planeta Ragnarel, el amor de ambos pudo más que cualquier distancia y civilización; sin embargo, la pareja tendrá que enfrentar aún más obstáculos como la venganza de la incomprensión y celos (Andina Noticias, 2017).

Las grandes dosis de aventura, las batallas espaciales y los enfrentamientos entre ejércitos siguen siendo un componente esencial de esta novela, así como las referencias socioculturales de las distintas especies que aparecen en la narración.

Según comenta el autor, en el ciclo de entrevistas realizadas por el Instituto Pedro Peralta y Barnuevo - Océano a escritores de novelas de space opera en el Perú durante el siglo XXI, sus principales influencias para la trilogía de Planetas perdidos, Años luz y Retorno a la Tierra son las narraciones de ciencia ficción que aparecieron, principalmente, entre las décadas de 1940 y 1960. Estos textos eran producidos como literatura de masas, al priorizar el contenido ante la forma y apuntar a grandes mercados de lectores interesados en tan solo pasar un buen rato (Instituto Océano, 2020b).

Sin embargo, el nombre Alderán de planeta al que Arthur llega en el primer libro parece ser una referencia directa al planeta Alderaan que aparece en la saga Star Wars y que es destruido por la Estrella de la muerte en el episodio IV.

Veinte mil años después, publicada en 2018, es otra de las novelas futuristas de Alfredo Dammert que se enmarca en el subgénero de la space opera. En esta novela el planeta Tierra se ha visto prácticamente destruido por diversas catástrofes que han puesto en riesgo la existencia de la humanidad. En este contexto, una raza de seres extraterrestres invade el planeta Tierra, con la apariencia de tener buenas intenciones al hacerse pasar por arcángeles, y reconstruyen la civilización humana a su antojo y para su propio beneficio. Así, se valen de la fe cristiana para manipular a la humanidad. Entre las disposiciones que imponen a los seres humanos, se encuentra la de prohibir la tecnología. Esto hace retroceder el desarrollo de la humanidad hasta casi retornar a la Edad Media. El protagonista de la novela nota la farsa de los extraterrestres y se enfrenta a ellos y a sus seguidores. A pesar de verse superado por sus enemigos, el protagonista logra contactarse con otros terráqueos que mucho tiempo atrás abandonaron la Tierra y logra escapar con ellos a otros mundos.

La novela, en sí, implica una crítica contra lo que se establece en forma de imposición. En ese sentido plantea el no aceptar a rajatabla las disposiciones de la autoridad, si estas no se encuentran fundamentadas en la razón y el beneficio social. 
El hecho de que los extraterrestres utilizaran la religión cristiana para enmascarar su manipulación resulta interesante en cuanto también establece la posibilidad de crítica y revisión frente a la fe, lo cual implica una tendencia progresista en su planteamiento.

La representación de estos extraterrestres que se hacen pasar por arcángeles también implica un quiebre con los estándares establecidos, pues estos supuestos arcángeles son descritos con características similares a demonios.

Según comenta el autor, en el ciclo de entrevistas realizadas por el Instituto Pedro Peralta y Barnuevo - Océano a escritores de novelas de space opera en el Perú durante el siglo XXI, al escribir esta novela encontró cierta influencia en el mito moderno de los "anunakis", el cual plantea que la especie humana se encuentra dominada por una raza extraterrestre de humanoides reptilianos (Instituto Océano, 2020b); idea muy extendida en la cultura popular moderna, principalmente a la vinculada con lo que llaman las "teorías de los antiguos astronautas", las cuales sugieren que la especie humana, desde tiempos inmemoriales, ha sido dirigida por extraterrestres. Estos planteamientos son masivamente difundidos en diversos programas de radio, televisión y medios alternativos, que incluyen las transmisiones por redes sociales y YouTube.

Reina del espacio, publicada en 2018, es la historia de un joven peruano, llamado Bernardo, quien, cuando niño, descubre junto a un tío una nave espacial en una caverna. Dentro de la nave encuentran a unos seres antropomorfos muertos. Esto lo impacta y lo motiva a estudiar astrofísica, para lo cual viaja a Estados Unidos. Termina por trabajar en la NASA y descubre que los seres que encontró en la caverna cuando niño están volviendo a la Tierra. Estos seres empiezan a perseguirlo y, al notarlo, cae en la cuenta de que su vida corre riesgo. En su escape vive distintas aventuras y llega a la India, donde obtiene el conocimiento necesario para desdoblarse y viajar incorpóreamente.

Luego de aprender lo necesario sobre el viaje incorpóreo, Bernie, como lo llaman desde su estadía en Estados Unidos, se traslada a Alemania, donde, con ayuda de científicos, construye una máquina que le permite viajar, de manera incorpórea, a otros planetas. Gracias a esta máquina se entera de que los extraterrestres que lo persiguen se dirigen a la Tierra y que han buscado invadirla desde hace mucho, pero fueron repelidos por seres antiguos, vinculados a los dioses primordiales de otro plano dimensional. Con el fin de combatir a estos extraterrestres, Bernie viaja a distintos planetas. Allí genera alianzas y brinda su apoyo y 
conocimiento para enfrentar a los extraterrestres malignos que avanzan hacia la Tierra e invaden todos los planetas en su camino.

Los mundos por los que Bernie viaja son diversos e incluso uno es representado como el reino de las amazonas, un planteamiento recurrente en el imaginario de ciencia ficción vinculado a la space opera. Ejemplos de esto se dan en ficciones como Flash Gordon, Star Trek, el universo Marvel, entre otros. La representación de la sociedad de los extraterrestres enemigos es planteada como dictatorial, militarizada; arquetipos malignos también vinculados a la tradición de la space opera.

La presencia de seres antiguos, semidioses o dioses, quienes contribuyen a enfrentarse a los extraterrestres invasores, tampoco es ajena a las narrativas de space opera. Como ejemplo, se puede considerar a la saga Stargate, en la que unos seres llamados "antiguos", que pueden moverse en distintos planos dimensionales, ayudan a los terrícolas a enfrentarse a seres malignos de civilizaciones también muy antiguas dispuestas a imponer su dominio en el cosmos o alimentarse de los seres humanos.

Según comenta el autor, en el ciclo de entrevistas realizadas por el Instituto Pedro Peralta y Barnuevo - Océano a escritores de novelas de space opera en el Perú durante el siglo XXI, sus influencias para esta novela se vinculan con los libros de ciencia ficción, principalmente los de space opera norteamericanas que ha leído. Relaciona esto con las grandes batallas espaciales, la presencia de científicos y el personaje con características idealistas y soñadores que esperan viajar a otros mundos. A esto se suma su referencia a las creencias indias respecto al desdoblamiento y la posibilidad de los viajes astrales, ideas muy difundidas en todo el mundo durante la segunda mitad del siglo XX. También se inspiró un poco en las teorías de los "antiguos astronautas", principalmente las vinculadas a que seres extraterrestres llegaron a la Tierra para salvarla de algún mal superior (Instituto Océano, 2020b).

\section{Influencia de Star Wars y la cultura popular norteamericana de finales del siglo XX}

En este grupo encontramos autores cuya principal influencia es la cultura popular norteamericana, encabezada por la saga de ciencia ficción Star Wars.

Empezaremos comentando los libros de Luis T. Moy: Alex Gubbins y los piratas del espacio (2010) y Alex Gubbins y el planeta olvidado (2013). Como resulta obvio, el segundo de los libros mencionados se trata de la continuación del primero y trae como novedad el crecimiento de Alex. Según el autor, ambos libros 
recurrieron a tópicos de la cultura de masas, principalmente del cine y la televisión, como elementos de su narrativa (Instituto Océano, 2020c).

El primer libro, publicado por la editorial Arkabas en 2010, cuenta cómo luego de que sus padres se separaran de él para salvarlo y ser rescatado por un militar galáctico, Alex Gubbins es entrenado en una academia militar donde aprende distintas habilidades para la batalla. A pesar de lo que podría esperarse, esta academia no se muestra como un lugar difícil y violento, como sucede en la mayoría de narrativas respecto a espacios de educación castrense, sino que es un espacio de encuentro y confraternidad. Respecto a esto, Elton Honores nos dice lo siguiente:

Es una visión amable: los cadetes del primer año reciben aplausos de los mayores, una banda de músicos androides toca para ellos, hay bebidas y bocaditos, en su primera jornada (Honores, 2017, p. 149).

Es posible que el enfoque del libro, dirigido a un público infantil-juvenil, haya determinado esta especie de relaciones "amables". Sin embargo, los libros de esta saga no dejan de lado distintos elementos que nos anclan con la realidad contingente, como las diferencias sociales, la pobreza y el crimen. En el primer libro incluso aparecen androides que cumplen el rol de piratas.

La influencia de la cultura de masas es notoria desde este primer volumen, que termina con una batalla espacial, muy representativa del space opera, sobre la cual Elton Honores dice lo siguiente:

La última escena de la novela está notablemente influenciada por las acciones de combate de Star Wars (Honores, 2017, p. 150).

Star Wars representa, tal vez, el arquetipo básico de la cultura popular globalizada y globalizante, el modelo dominante, como bien dice Honores. Según comenta el autor Luis T. Moy, en el ciclo de entrevistas realizadas por el Instituto Pedro Peralta y Barnuevo - Océano a escritores de novelas de space opera en el Perú durante el siglo XXI, esta saga de películas es una influencia directa para ambas novelas (Instituto Océano, 2020c).

La segunda novela, Alex Gubbins y el planeta olvidado, publicada esta vez bajo el sello de Ediciones Altazor en 2013, cuenta cómo el protagonista continúa su búsqueda por encontrar a sus padres y, en este camino, se dirige a el planeta olvidado: la Tierra, dejada de lado por la humanidad mucho tiempo atrás (tópico clásico de la ciencia ficción utilizado por Asimov en su saga Fundación y por 
muchos otros escritores, incluso otros peruanos, como Carlos de la Torre Paredes, quien también menciona una búsqueda del planeta Tierra en su saga Herederos del cosmos, y Carlos Echevarría, quien, en su saga El planeta olvidado, también llama "el planeta olvidado" a la Tierra).

Las relaciones sociales entre las distintas especies no generan gran conflicto en estos dos libros. A pesar de tratarse de especies distintas (humanos que interactúan con alienígenas), la cultura que muestran resulta homogénea, salvo por las diferencias físicas que representan a cada una de estas especies. Este planteamiento homogeneizante de las sociedades podría deberse a la condición globalizada y globalizante que parece implícita en el subgénero de la space opera, y que puede resultar una herramienta útil al momento de configurar cuerpos políticos inmensos y bien organizados.

Carlos Echevarría es otro de los autores que encuentran clara influencia en la cultura popular norteamericana de finales del siglo XX. Su saga de El planeta olvidado, a la fecha, cuenta con cuatro publicaciones de cinco previstas (Instituto Océano, 2020d). Tres de una tetralogía y una precuela que busca otorgarles un marco histórico a las aventuras narradas desde el primer libro El planeta olvidado 1: La liberación.

Hay un diseño de personajes más consistente, sobre todo en las descripciones físicas y en ciertas acciones de estrategia militar. La segunda mitad de la novela son acciones de guerra propiamente que insertan a esta novela dentro de la space opera, con claras influencias del cine de Star Wars o Starship Troopers, ya que algunas escenas son narradas con estilo cinematográfico (Honores, 2017, p. 151).

En El planeta olvidado 1: La liberación, que se publicó en 2012 bajo el sello de Editorial San Marcos, la Tierra, que es "el planeta olvidado" por el resto del cosmos, en el mundo posible creado por Echevarría, es anexado a la Federación Organizada del Universo Descubierto (FOUD), un cuerpo político democrático interplanetario que traerá diversos beneficios a la humanidad, principalmente relacionados con ciencia y tecnología. Durante este proceso se selecciona a un total de 12 representantes de la Tierra, entre los que se encuentra el protagonista, Fernando Villanueva, quien, al principio de la novela, se muestra dubitativo e indeciso respecto a qué hacer con su vida, pero mientras la historia avanza, entra en un proceso de autodescubrimiento que le permite darse cuenta de qué es capaz y qué quiere realmente. 
Debido a la anexión de la Tierra a la FOUD, la humanidad se involucra en la guerra que la FOUD mantiene contra el Imperio toriano, otro cuerpo político con gran influencia militar en el universo conocido. La trama gira según esta guerra y a cómo Fernando Villanueva y los demás terrícolas la enfrentan. Resultan importantes los procesos de desarrollo y asimilación de las nuevas condiciones que se establecen en la Tierra, pues involucran relaciones políticas y diplomáticas que cambian la realidad terrestre en poco tiempo.

El planeta olvidado 2: La resistencia, publicado en 2016 bajo el sello de Torre de Papel Ediciones, mantiene la lógica narrativa del primer libro, que basa su estética en las grandes batallas espaciales (al estilo de Star Wars) y las tramas políticas. En este caso el planeta Tierra está más involucrado con la FOUD, lo que trae complicaciones y tensiones entre las naciones terrestres, que pugnan por, cada una, poner a su propio representante frente a la federación interplanetaria. Además, las tropas del Imperio toriano avanzan hacia las galaxias centrales de la FOUD a fin de conquistarla. Por su parte, Fernando Villanueva, el protagonista de la saga, continúa en su camino de crecimiento para convertirse en un héroe. Las relaciones sociales y culturales en esta novela están marcadas por la interrelación entre la humanidad y la alianza interplanetaria que le brinda posibilidades tecnológicas para seguir superando diversos problemas. Las tensiones que se generan respecto a la FOUD están principalmente relacionadas con la guerra a la que arrastran a la humanidad como parte de la alianza.

El planeta olvidado 3: La odisea, publicado en 2019 bajo el sello de Torre de Papel Ediciones, es, tal vez, el libro que, estructural y narrativamente, se diferencia más de la saga. Esto, en parte, porque, a diferencia de los otros dos libros, no incide tanto en las grandes batallas espaciales, sino que más bien se enfoca en las peripecias que vive su protagonista para regresar al planeta Tierra. El hecho de que su título sea La odisea no resulta gratuito, pues hace una referencia directa al clásico de la literatura universal, en la que el personaje de Ulises, enfrentándose a un sinfín de peligros y obstáculos, busca volver a casa (Instituto Océano, 2020d). En esta novela de Echevarría, su personaje, Fernando, que ha sido tomado prisionero por el Imperio toriano, escapa de su cautiverio e inicia su viaje de vuelta a casa. En esta oportunidad, el autor pone énfasis en el desarrollo del mundo interior del personaje principal, lo que contribuye a mostrar un fuerte contraste respecto al personaje que encontramos en la primera novela de la saga. En este sentido, el viaje de retorno al planeta Tierra es, a la vez, un viaje de autodescubrimiento de Fernando. 
La galaxia escarlata, publicada en 2015 por Torre de Papel Ediciones, se trata de la precuela de la saga de Echevarría. Esta novela narra el enfrentamiento entre las dos fuerzas más poderosas del universo conocido: la FOUD y el Imperio toriano, cada bando liderado por un poderoso y carismático héroe. El presidente Jorleff, de la FOUD, liderará los ejércitos de la federación, mientras que el Imperio toriano tiene a la cabeza al emperador Osturus Cruldestor, quien pretende conquistar todo el universo.

Respecto a esta novela, Elton Honores nos dice lo siguiente:

Aquí los buenos son buenos y los malos son malos. No hay término medio, a diferencia del mundo real, en el que hay espacio para lo gris, para aquello que no se define aún o termina de definirse.

La novela de Echevarría se inserta dentro de la space opera. Contiene todos los elementos tópicos: los piratas espaciales —al inicio de la novela-, el racismo (las diferentes razas que pueblan el universo), las batallas espaciales y la política colonial. La FOUD promueve una economía basada en el comercio (con una serie de redes burocrático-administrativas), mientras que los torianos constituyen una fuerza militar que simplemente quiere gobernar todo el universo (Honores, 2016).

Según comenta Carlos Echevarría, en el ciclo de entrevistas realizadas por el Instituto Pedro Peralta y Barnuevo - Océano a escritores de novelas de space opera en el Perú durante el siglo XXI, sus influencias al momento de crear historias vienen, en gran medida, de la cultura de masas, lo que incluye al cine, la literatura, los videojuegos, los cómics y las series de televisión (Instituto Océano, 2020d).

Carlos de la Torre Paredes es otro de los autores que encuentran su principal influencia en la cultura popular norteamericana de finales del siglo XX. Su saga Herederos del cosmos cuenta con dos novelas y, además, es autor de tres cuentos en el libro Ruffus tiene finura y otros cuentos igual de selectos; uno de ellos, el cuento "SOS", fue publicado de forma individual en 2016. Para fines de esta investigación, solo nos enfocaremos en las novelas Los viejos salvajes y La conquista de Piro. Si bien ambas novelas forman parte de la saga Herederos del cosmos, no se encuentran relacionadas entre sí. Incluso, argumental y estéticamente, se diferencian sobremanera.

Empezaremos por comentar un poco respecto de Los viejos salvajes, novela finalista del Concurso de Novela Breve Cámara Peruana del Libro 2012 y publicada 
el mismo año por Peithos Editores. Esta fue la primera novela que apareció de la saga, aunque la idea de saga surge recién con su segunda edición, esa vez con la editorial peruana La Nave, en la que al título se le agregó el subtítulo de Herederos del cosmos. Esta novela, además de establecerse dentro del space opera, también tiene grandes acercamientos al terror espacial, con referencias claras a Alien, el octavo pasajero de Ridley Scott. En sí, la novela cuenta cómo una raza alienígena antropofágica y con poderes telepáticos se apodera de una gran nave espacial humana. Entre sus principales características está el que sus protagonistas son todos adultos mayores que, de una forma u otra, añoran su pasado. La estética de la novela apunta a los ambientes oscuros y opresivos del espacio, y recurre a elementos de los videojuegos que se amalgaman con la narración, como el contar a los enemigos caídos o las referencias a los niveles de las naves y las armas.
A nivel formal la novela se construye sobre la base de la estética del videojuego, con sus victorias que se van acumulando y contando, como puntos o créditos para seguir subsistiendo, y los seres monstruosos al- teridad-oponente a los que hay que vencer. La vida es entendida como un videojuego en el que hay que matar para sobrevivir. (Honores, 2017, p. 158).

Sin embargo, los recursos narrativos también son tomados de otros exponentes de la cultura popular actual, como Star Wars y Starship troopers. Al respecto, Elton Honores señala:

se inscribe dentro del subgénero de la space opera, con guiños a films como Star Wars, Starship Troopers o Alien (Honores, 2017, p. 156).

La conquista de Piro, publicada por Torre de Papel Ediciones en 2019, a diferencia de Los viejos salvajes, no es una novela de terror espacial, sino que narra la historia de una conquista militar por parte de la Federación Latina al sistema planetario Piro, que se encontraba en una zona desconocida del cosmos, pero que alojaba humanos, también herederos de las tradiciones terrestres. Esta novela hace clara referencia a la conquista del Perú y traslada sucesos muy similares a los acontecidos en el archivo histórico a un escenario futurista, en el cual los seres humanos abandonaron el planeta Tierra miles de años antes. Momentos conocidos de la historia aparecen en la novela, e incluso empieza con una escena muy parecida a la que ocurre en la famosa isla del Gallo, donde Francisco Pizarro 
marca una línea en el suelo y ofrece riqueza a quien lo siguiera. Esta novela se encuentra repleta de enfrentamientos espaciales y de infantería sobre naves espaciales y planetas. Las referencias a Star Wars vuelven a ser claras. Por ejemplo, algunas de las estaciones espaciales de los quetua (los habitantes del sistema Piro) tienen tamaños descomunales, tanto que aparentan ser satélites de algún planeta; un claro homenaje a la famosa Estrella de la Muerte de Star Wars. Además, según el autor (Instituto Océano, 2020e), las batallas espaciales toman de ejemplo los planteamientos de distintos videojuegos.

\section{Influencia del anime japonés y la cultura popular alternativa de finales del si- glo XX}

Este último grupo de autores, al igual que los anteriores, bebe de muchas fuentes, pero encuentra su característica diferenciadora al recurrir a elementos de la cultura popular alternativa de finales del siglo $\mathrm{XX}$, principalmente el anime japonés.

Tomás Béjar, con su libro Y deus dijo (2015), publicado como edición de autor, y en un primer momento de forma virtual por la plataforma Amazon, nos narra una historia ambientada en el año 2237 d. C. Esta novela trata del viaje de un equipo liderado por la doctora Hira Tanaka hacia Ofelia, el satélite de Urano, a fin de llegar a la Biblioteca Mentor, donde se guarda el conocimiento de toda la humanidad antes de que fuera arrasada por el impacto del cometa Halley. En la misma carrera se encuentra el doctor Arquímedes Amaru, quien, junto con Boris Vallejo y otros secuaces, se enfrenta a la sociedad de Nuevo Mundo, que se basa en el autoritarismo para traer orden a lo que ha quedado de la humanidad. Estos personajes, que en un primer momento se perfilan como los antagonistas, terminarán por influir significativamente en la trama. La mención a los nombres Amaru y Vallejo no es gratuita. Según comenta el autor, en el ciclo de entrevistas realizadas por el Instituto Pedro Peralta y Barnuevo - Océano a escritores de novelas de space opera en el Perú durante el siglo XXI, él decidió utilizarlos para vincular su obra a la historia del Perú (Instituto Océano, 2020f). La sociedad descrita en la novela corresponde a una teocracia tecnológica y autoritaria, que utiliza el bienestar paternalista como excusa para imponerse por la coerción e intenta someter a diversos grupos y tribus periféricas a su civilización. Estas tribus, que representan un mundo salvaje y libre frente a la civilización impositiva de Nuevo Mundo, guardan cierta similitud con las tribus de la saga de películas Mad Max. Respecto a sus influencias, Elton Honores dice que la novela es: 
De estilo visual [...] toma elementos de la tradición cinematográfica de modo claro, desde Alien y Star Wars, hasta filmes más recientes como Matrix y Sector 9 (Honores, 2017, p. 160).

Sin embargo, según el autor, su influencia está relacionada con textos como 2001. Una odisea espacial y el anime japonés, lo cual se muestra claramente con las batallas de grandes robots (Instituto Océano, 2020f).

Jim Rodríguez es otro autor que encuentra sus principales influencias en la cultura popular alternativa de finales del siglo XX. Su novela Apokbalipzis. La máquina del tiempo (2015) trata el conflicto entre el Imperio Anexa y la Unión, que son dos conglomerados de sistemas planetarios. En medio de este conflicto aparece un ser poderoso llamado Apokhalipzis, un destructor de mundos que se alimenta de la energía del núcleo de los planetas, un claro homenaje al personaje Galactus del universo de Marvel. Mientras tanto, en el planeta Tierra, la protagonista, Kate Castel, crea una máquina del tiempo como parte de su trabajo para los militares. Cuando Apokhalipzis llega a la Tierra se entera de la existencia de esta máquina y decide hacerse con ella. Respecto a esta novela, Elton Honores comenta lo siguiente:

La novela se divide en dos partes. La primera está dominada por la space opera (los piratas espaciales, el racismo - las diferentes razas que pueblan el universo-, las batallas espaciales y la política colonial) [...] La novela da un giro hacia la mitad cuando se introduce la máquina del tiempo y el universo terrestre, lo cual es un acierto dentro de la narración (Honores, 2017, pp. 159-160).

Respecto a sus influencias, el escritor Benjamín Román, también citado por Elton Honores, comenta:

Sus líneas también nos llevan a deducir algunas de las fuentes que las alimentan: los cómics y los dibujos animados, incluyendo el manga. Por ejemplo, podemos percibir la influencia de la factoría Marvel y su fuerza cósmica, Galactus. También de Dragon Ball, sobre todo en la transformación de los personajes en seres cada vez más poderosos (Román, 2015).

Respecto a esto, el autor, en el ciclo de entrevistas realizadas por el Instituto Pedro Peralta y Barnuevo - Océano a escritores de novelas de space opera en el 
Perú durante el siglo XXI, confirma que sus principales influencias son televisivas y cinematográficas, lo cual confirma lo planteado por Benjamín Román. Según sus propias palabras, Rodríguez es un "escritor que capta de forma más visual" y lo relaciona a una tendencia generacional (Instituto Océano, 2020g).

\section{Conclusiones}

Estas 16 novelas de siete distintos autores son algunos ejemplos de la space opera producida en el Perú durante el siglo XXI. Pueden servir de muestra referencial de la producción que se realiza en este subgénero de la ciencia ficción, ya que configuran algunas de sus principales características. Tal vez la más resaltante sea que la space opera peruana producida durante el siglo XXI no deja de lado la tradición del subgénero de mantener como fuente la cultura de masas, si bien con distintos matices, determinados por la edad de los autores, la época en la que consumieron cultura de masas y sus preferencias específicas. Ello nos permitió enmarcarlos en los tres grupos desarrollados anteriormente.

De todo lo expuesto se puede concluir que las novelas de space opera producidas en el Perú durante el siglo XXI están fuertemente influenciadas por la cultura de masas de distintas épocas, que parece coincidir con las etapas de juventud y adolescencia de los autores y configura una suerte de producción generacional. También se puede concluir que estas influencias se relacionan con productos culturales de difusión global (cultura de masas) que se presentaron en diversas plataformas expresivas que van desde cuentos y novelas, historietas, novelas gráficas y caricaturas, series de televisión, películas de cine, hasta, más recientemente, videojuegos, documentales sensacionalistas en canales de cable y contenido de redes sociales y de youtubers; esto ya había sido mencionado por Elton Honores. También queda claro que la tendencia de trabajar la space opera en formato de sagas está presente en gran parte de la producción peruana del siglo XXI, tal como comenta David Olier. Esto podría hacer eco con la tendencia global del space opera de producirse en sagas, a fin de maximizar las ventas de las editoriales.

\section{Contribución del autor}

Carlos Andrés de la Torre Paredes ha participado en la elaboración, la compilación de datos, la redacción y el consentimiento de la versión final del presente artículo. 


\section{Fuente de financiamiento}

La investigación es autofinanciada.

\section{Conflictos de interés}

Ninguno.

\section{Trayectoria académica}

Carlos Andrés de la Torre Paredes es licenciado en Ciencia Política por la Universidad Nacional Federico Villarreal y egresado de la maestría en Escritura Creativa de la Universidad Nacional Mayor de San Marcos. Ha publicado los libros Los viejos salvajes (2012), El señor de la guerra. Campos de batalla (2014), Ruffus tiene finura y otros cuentos igual de selectos (2018) y La conquista de Piro (2019).

\section{Referencias bibliográficas}

Andina. (28 de abril de 2017). Peruano presentará trilogía de ciencia ficción en Feria del Libro de Buenos Aires. Correo. https://andina.pe/agencia/noticia-peruano-presentara-trilogia-ciencia-ficcion-feria-del-libro-buenos-aires-664815.aspx

Anónimo. (11 de enero de 2017). Alfredo Dammert: Presentan Años luz, libro de ciencia ficción de escritor peruano. Correo. https://diariocorreo.pe/cultura/alfredo-dammert-presentan-anos-luz-libro-de-ciencia-ficcion-de-escritor-peruano-723482/

Asimov, I. (1999). Sobre la ciencia ficción. Editorial Sudamericana.

Clute, J., Nicholls, P., Langford D. y Sleight G. (13 de enero de 2020). Space Opera. The Encyclopedia of Science Fiction. http://sf-encyclopedia.com/entry/space_opera

Cuauh. (8 de febrero de 2011). Space Opera [entrada de blog]. Café Anime Lair. https://cafeanimelair.com/2011/02/08/space-opera/

Honores, E. (13 de enero de 2016). Jim Rodriguez: Apokhalipzis. La máquina del tiempo [blog]. Iluminaciones. https://eltonhonores.blogspot.com/2016/01/

Honores, E. (2017). La división del laberinto. Estudios sobre la narrativa fantástica peruana contemporánea (1980-2015). Polisemia.

Instituto Pedro Peralta y Barnuevo -. Océano (9 de octubre de 2020a). Entrevista a Adriana Alarco de Zadra. Novelas de space opera en el Perú durante el siglo XXI [video].https://youtu.be/wlNftbXQ7Z0 
Instituto Pedro Peralta y Barnuevo - Océano. (3 de octubre de 2020b). Entrevista a Alfredo Dammert. Novelas de space opera en el Perú durante el siglo XXI [video]. https://youtu.be/BNIYZXeoexI

Instituto Pedro Peralta y Barnuevo - Océano. (9 de octubre 2020c). Entrevista a Luis T. Moy. Novelas de space opera en el Perú durante el siglo XXI [video]. https:// youtu.be/K1p_OG_iAV0

Instituto Pedro Peralta y Barnuevo -. Océano. (8 de octubre de 2020d). Entrevista a Carlos Echevarría. Novelas de space opera en el Perú durante el siglo XXI [video]. https://youtu.be/g43BGbuP4HU

Instituto Pedro Peralta y Barnuevo -. Océano. (25 de octubre de 2020e). Entrevista a Carlos de la Torre Paredes. Novelas de space opera en el Perú durante el siglo XXI [video].https://youtu.be/cwqdcemhcsc

Instituto Pedro Peralta y Barnuevo -. Océano. (7 de octubre de 2020f). Entrevista a Tomás Béjar. Novelas de space opera en el Perú durante el siglo XXI [video]. https://youtu.be/1MTYZ70PSyQ

Instituto Pedro Peralta y Barnuevo -. Océano. (24 de agosto de 2020g). Entrevista a Jim Rodríguez. Novelas de space opera en el Perú durante el siglo XXI [video]. https://youtu.be/beV0amQ7erk

Jameson, F. (2009). Arqueologias del futuro. El deseo llamado utopia y otras aproximaciones de ciencia ficción. Ediciones Akal.

Mann, G. (2001). The Mammoth Encyclopedia of Science Fiction. Robinson, an imprint of Constable \& Robinson Ltd.

Olier, D. (20 de abril de 2020). Las mejores óperas espaciales [entrada de blog]. El rincón de Cabal. https://cabaltc.com/mejores-operas-espaciales

Roman, B. (octubre de 2015). Apokhalipzis, la máquina del tiempo. Jim Rodríguez reseña por Benjamín Román Abram [entrada de blog]. Más que imaginar. https:// masqueimaginar.blogspot.com/2015/10/apokhalipzis-la-maquina-del-tiempo-jim.html

Rothgiesser, H. (6 de noviembre de 2015). Alfredo Dammert, Planetas perdidos [entrada de blog]. Mildemonios Cultural. https://mildemonioscultural.wordpress. com/2015/11/06/alfredo-dammert-planetas-perdidos/ 